\title{
Nuclear data for medical applications: An overview of present status and future needs
}

\author{
Syed M. Qaim ${ }^{\mathrm{a}}$ \\ Institut für Neurowissenschaften und Medizin, INM-5: Nuklearchemie, Forschungszentrum Jülich, 52425 Jülich, Germany
}

\begin{abstract}
A brief overview of nuclear data required for medical applications is given. The major emphasis is on radionuclides for internal applications, both for diagnosis and therapy. The status of the presently available data is discussed and some of the emerging needs are outlined. Most of the needs are associated with the development of non-standard positron emitters and novel therapeutic radionuclides. Some new developments in application of radionuclides, e.g. theranostic approach, multimode imaging, radionanoparticles, etc. are described and the related nuclear data needs are discussed. The possible use of newer irradiation technologies for medical radionuclide production, e.g. intermediate energy charged-particle accelerators, high-power electron accelerators for photon production, and spallation neutron sources, will place heavy demands on nuclear data.
\end{abstract}

\section{Introduction and general overview}

Nuclear data are needed for applications in many fields. The scope of this article is limited to medical applications. The basic aim of the related data is to provide a fundamental base for external radiation therapy and, more importantly, for optimum production and internal application of radionuclides. The activities consist of new experimental measurements, nuclear model calculations, and standardisation and evaluation of existing data [cf. 1].

The most commonly used modality in the radiation treatment of a patient is photon therapy which is carried out using $\gamma$-rays from a ${ }^{60} \mathrm{Co}$ source or harder $\gamma$-rays from a linear accelerator. Since the photon energies are below the threshold of a nuclear reaction, the nuclear data play almost no role and atomic and molecular data are much more important [cf. 2]. The radiation causes ionisation and excitation of atoms and molecules and it is mostly the locally generated secondary electrons that lead to the therapeutic effect. Another modality is fast neutron therapy. The nuclear data requirements for this modality are extensive, especially with regard to (n, charged particle) reactions induced in the tissue. It is these secondary charged particles (i.e. protons and ${ }^{4} \mathrm{He}$ nuclei) which lead to the therapeutic effect. In recent years, however, due to its unspecific nature the neutron therapy has been mostly abandoned. A newer fast developing modality involves the use of 70 to $250 \mathrm{MeV}$ protons. As is well known, the protons lose energy while traversing the tissue but their intensity loss is mainly towards the end of the path length, i.e. in the "Bragg peak" area. This makes the proton therapy very specific. It should, however, be pointed out that also in proton therapy atomic and molecular data are of prime importance and extensive investigations are underway in several laboratories. Those studies are, however, beyond the scope of this review. As

a e-mail: s.m.qaim@fz-juelich.de far as nuclear data are concerned, non-elastic cross sections as well as microscopic cross sections, average energies and angular distributions of emitted $\mathrm{n}, \mathrm{p}, \mathrm{d}, \alpha$ and $\gamma$ are of interest [cf. 3]. The data on the formation of activation products are also needed, although it has been shown that the dose in proton therapy due to radioactive products is $<1 \%$ of the total dose [4]. In a rather specialized form of charged-particle therapy, heavy-ion beams are used. The status and needs of data are somewhat similar to those for proton therapy. Charged-particle-therapy - related nuclear data work is performed in a few special laboratories, some of which is being reported at this conference.

As far as internal applications of radionuclides are concerned, both diagnosis and therapy are performed [cf. 5]. Each application, however, demands a special type of radionuclide, its selection being dependent on its decay data. There are two guiding factors

a) efficient detection of emitted radiation from outside of the body,

b) radiation dose caused to the patient.

For efficient detection, a short-lived radionuclide emitting a single or major $\gamma$-ray of energy about $150 \mathrm{keV}$ or a positron is very suitable. Regarding the radiation dose, attention has to be paid to the emitted corpuscular radiation, i.e. $\alpha$ or $\beta^{-}$particles (or low-energy electrons). A complete set of decay data is needed to calculate the radiation dose.

The production of a radionuclide demands a knowledge of nuclear reaction cross sections. These data are important to

(i) determine the optimum energy range for the production of the desired radionuclide,

(ii) calculate the expected thick target yield of the radionuclide,

(iii) determine the level of isotopic radionuclidic impurities for a given thickness and enrichment of the target material. 
In a nuclear reactor, radionuclide production is generally done via nuclear fission or an $(n, \gamma)$ reaction. In a few special cases double neutron capture and $(n, p)$ reactions are also used. In general, neutron-spectrum averaged cross sections of all those processes are available, though with relatively large uncertainties in a few cases. In cyclotron production of a radionuclide the data requirements are much more stringent than in a nuclear reactor. A full knowledge of the excitation functions of the relevant nuclear reactions is needed.

This article gives an overview of the nuclear data needed for medical applications, mainly those related to internal applications of radionuclides. The emphasis is on data for novel radionuclides, where the needs are extensive and often demanding.

\section{Commonly used radionuclides in nuclear medicine}

\subsection{Diagnostic radionuclides}

The underlying principle in diagnostic nuclear medicine is that the radiation dose to the patient is as low as possible, compatible with the required quality of imaging and the diagnostic advantage in comparison to nonradioactive methods. To this end, in Single Photon Emission Computed Tomography (SPECT), $\gamma$-ray emitting radionuclides like ${ }^{99 \mathrm{~m}} \mathrm{Tc}\left(\mathrm{T}_{1 / 2}=6.0 \mathrm{~h}\right),{ }^{123} \mathrm{I}\left(\mathrm{T}_{1 / 2}=13.2 \mathrm{~h}\right)$ and ${ }^{201} \mathrm{Tl}\left(\mathrm{T}_{1 / 2}=3.06 \mathrm{~d}\right)$ are commonly used, and to some extent also ${ }^{67} \mathrm{Ga}\left(\mathrm{T}_{1_{2}}=3.2 \mathrm{~d}\right)$ and ${ }^{111} \mathrm{In}\left(\mathrm{T}_{1_{2}}=2.8 \mathrm{~d}\right)$. However, the latter two are considered today also useful for Auger therapy. In Positron Emission Tomography (PET), on the other hand, short-lived $\beta^{+}$-emitting radionuclides like ${ }^{11} \mathrm{C} \quad\left(\mathrm{T}_{1 / 2}=20.4 \mathrm{~min}\right),{ }^{13} \mathrm{~N} \quad\left(\mathrm{~T}_{1 / 2}=10.0 \mathrm{~min}\right),{ }^{15} \mathrm{O}$ $\left(\mathrm{T}_{1 / 2}=2.0 \mathrm{~min}\right), \quad{ }^{18} \mathrm{~F} \quad\left(\mathrm{~T}_{1 / 2}=110 \mathrm{~min}\right), \quad{ }^{68} \mathrm{Ga} \quad\left(\mathrm{T}_{1 / 2}=\right.$ $68.3 \mathrm{~min})$ and ${ }^{82} \mathrm{Rb}\left(\mathrm{T}_{1 / 2}=1.3 \mathrm{~min}\right)$ often find application. The radionuclides ${ }^{99 \mathrm{~m}} \mathrm{Tc},{ }^{68} \mathrm{Ga}$ and ${ }^{82} \mathrm{Rb}$ are available through generator systems. The parent of ${ }^{99 \mathrm{~m}} \mathrm{Tc}$ (i.e. ${ }^{99} \mathrm{Mo}$ ) is produced in a nuclear reactor but the parents of ${ }^{68} \mathrm{Ga}$ and ${ }^{82} \mathrm{Rb}$ (i.e. ${ }^{68} \mathrm{Ge}$ and ${ }^{82} \mathrm{Sr}$ ) are produced using intermediate energy protons from a cyclotron or an accelerator. All the other radionuclides are produced via direct charged-particle induced reactions at cyclotrons. Except for a few discrepancies, the decay and production data of all those routinely used radionuclides are well known [cf. 6-8]. However, if any of the presently used SPECT radionuclides is to be considered for application in Auger therapy, more detailed Auger electron spectra would be needed.

In recent years an apprehension has arisen about the shortage in the supply of the most commonly used SPECTradionuclide ${ }^{99 \mathrm{~m}} \mathrm{Tc}$, due to possible shut-down of nuclear research reactors. This has led to considerable scientific effort with the aim to develop alternative accelerator-based production methods for the parent radionuclide ${ }^{99} \mathrm{Mo}$ as well as the desired product ${ }^{99 \mathrm{~m}} \mathrm{Tc}$. Nuclear reactions induced by fast neutrons, fast photons as well as $10-$ $40 \mathrm{MeV}$ protons and deuterons have been investigated (for recent reviews cf. $[9,10]$ ). Nuclear data for some reactions are known [cf. 11-13] with reasonable accuracy for present calculations. Today, the most promising route is the ${ }^{100} \mathrm{Mo}(\mathrm{p}, 2 \mathrm{n}){ }^{99 \mathrm{~m}} \mathrm{Tc}$ reaction using highly enriched target material. The cross section data for this reaction have been thoroughly measured [cf. 14-16] and evaluated [17], so that the database is now reliable. However, for a critical check of the impurities associated with this process as well as for development of other possible production routes, further extensive nuclear data studies are necessary.

\subsection{Therapeutic radionuclides}

In internal radionuclide therapy, a well-defined localized radiation dose needs to be deposited in a malignant or inflammatory tissue. Therefore, radionuclides emitting low-range highly-ionising radiation, i.e. $\alpha$ or $\beta^{-}$particles, conversion and/or Auger electrons, are of great interest. Among the $\beta^{-}$emitters, the radionuclides ${ }^{32} \mathrm{P}\left(\mathrm{T}_{1 / 2}=14.3 \mathrm{~d}\right),{ }^{89} \mathrm{Sr}\left(\mathrm{T}_{1 / 2}=50.5 \mathrm{~d}\right),{ }^{90} \mathrm{Y}$ $\left(\mathrm{T}_{1 / 2}=2.7 \mathrm{~d}\right),{ }^{131} \mathrm{I}\left(\mathrm{T}_{1 / 2}=8.0 \mathrm{~d}\right),{ }^{153} \mathrm{Sm}\left(\mathrm{T}_{1 / 2}=1.9 \mathrm{~d}\right){ }^{169} \mathrm{Er}$ $\left(\mathrm{T}_{1 / 2}=9.4 \mathrm{~d}\right),{ }^{177} \mathrm{Lu}\left(\mathrm{T}_{1 / 2}=6.7 \mathrm{~d}\right)$ and ${ }^{188} \operatorname{Re}\left(\mathrm{T}_{1 / 2}=17.0 \mathrm{~h}\right)$ are often used. The radionuclides ${ }^{90} \mathrm{Y}$ and ${ }^{188} \mathrm{Re}$ are available through generator systems whereas the others are produced directly or via decay of the respective precursors. Regarding $\alpha$-emitting radionuclides, hitherto ${ }^{211}$ At $\left(\mathrm{T}_{1 / 2}=7.2 \mathrm{~h}\right)$ and ${ }^{223} \mathrm{Ra}\left(\mathrm{T}_{1 / 2}=11.4 \mathrm{~d}\right)$ have found some application but several other radionuclides are in development (see below). As far as Auger electron and Xray emitters are concerned, so far only $\left.{ }^{125} \mathrm{I}_{\left(\mathrm{T}_{1 / 2}\right.}=59.4 \mathrm{~d}\right)$ and ${ }^{103} \mathrm{Pd}\left(\mathrm{T}_{1 / 2}=17.0 \mathrm{~d}\right)$, respectively, have found wide application.

The radionuclides ${ }^{211} \mathrm{At}$ and ${ }^{103} \mathrm{Pd}$ are produced at cyclotrons. All the other radionuclides are produced via irradiations in a nuclear reactor. Except for small discrepancies in some decay schemes or uncertainty in individual $(n, p),(n, \gamma)$ or double neutron capture cross sections, the decay and production data of all those radionuclides are fairly well known from the viewpoint of routine patient-care studies [cf. 8, 18, 19].

\section{Research oriented radionuclides}

In recent years, nuclear data research related to medical radionuclides has been carried out mainly on non-standard positron emitters and novel therapeutic radionuclides. A brief discussion is given below.

\subsection{Non-standard positron emitters}

With the growing significance of PET in diagnostic nuclear medicine, the need for novel positron emitters, also known as non-standard positron emitters, has been increasing, especially for studying slow metabolic processes and for quantification of targeted therapy. The decay data of the emerging positron emitters are

sufficiently known; yet discrepancies exist in several cases, especially with regard to the $\beta^{+}$emission intensities. It is therefore suggested to revise the mass decay chains of most of the potentially interesting positron emitters, and perform new measurements using improved techniques, wherever necessary.

The production data of non-standard positron emitters have been discussed in detail in recent reviews $[9,20]$. 


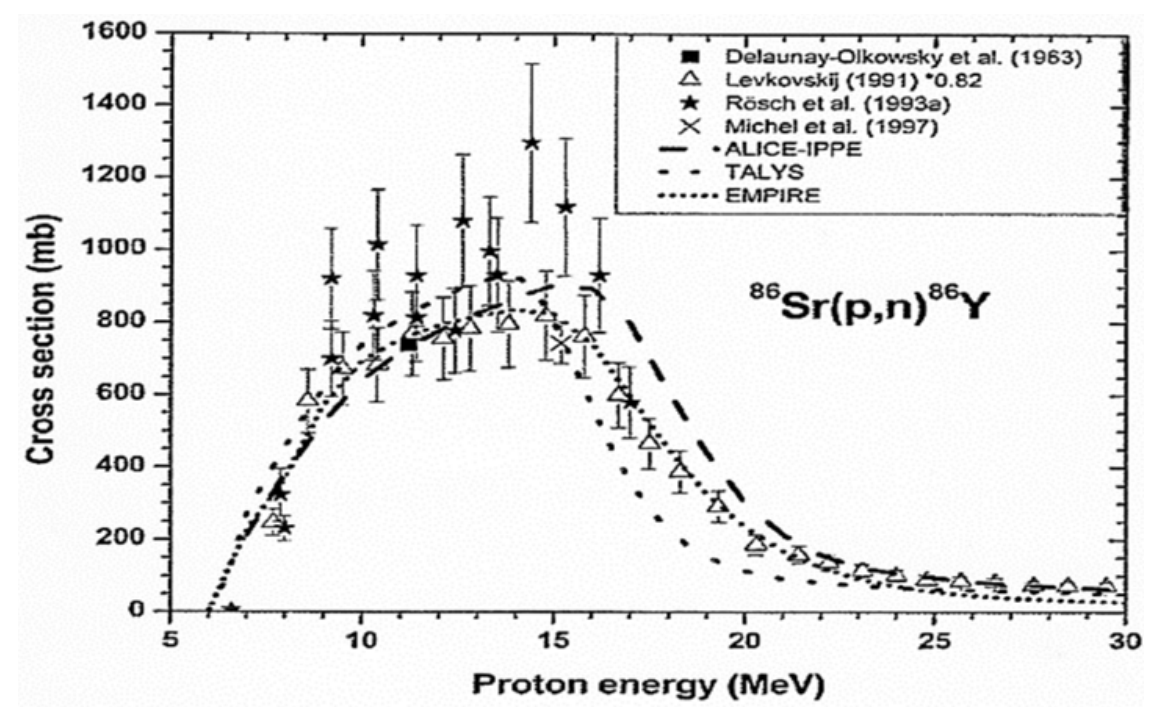

Figure 1. Normalised experimental data for the ${ }^{86} \mathrm{Sr}(\mathrm{p}, \mathrm{n}){ }^{86} \mathrm{Y}$ reaction along with the results of three nuclear model calculations, viz. ALICE-IPPE, TALYS and EMPIRE, plotted as a function of the proton energy (taken from Ref. [22]).

In this article, therefore, only a few salient features are considered. The development of a suitable production process of a novel positron emitting radionuclide demands investigation of several nuclear reactions. In most cases it is necessary to use a highly enriched isotope as target material. The choice of the production route then depends mainly on the yield of the desired radionuclide and the expected level of the radioactive impurities. So far about 25 novel positron emitters have been developed, most of them using a small-sized cyclotron (with $\mathrm{E}<20 \mathrm{MeV}$ ). The common route of production is the low-energy $(\mathrm{p}, \mathrm{n})$ reaction on an enriched target isotope. In a few cases other low-energy reactions, such as $(\mathrm{d}, \mathrm{n})$ and $(\mathrm{p}, \alpha)$, have also been employed [cf. 20]. Out of all the radionuclides developed, four of them, namely ${ }^{64} \mathrm{Cu},{ }^{86} \mathrm{Y},{ }^{89} \mathrm{Zr}$ and ${ }^{124} \mathrm{I}$, are finding wider application. The number of relevant reactions studied for their production has therefore also been correspondingly large. The low-energy $(p, n)$-reaction on an enriched target isotope is favoured because it generally leads to a high-purity product. For several radionuclides the $(\mathrm{p}, \mathrm{n})$ reaction data are now precisely known, e.g. for ${ }^{64} \mathrm{Cu}$ [cf. 21]. However, in several other cases there are inconsistencies. As a typical example, the excitation function of the reaction ${ }^{86} \mathrm{Sr}(\mathrm{p}, \mathrm{n}){ }^{86} \mathrm{Y}$ is shown in Fig. 1 [22]. The experimental data are compared with the results of nuclear model calculations using three standard codes, namely ALICE-IPPE, TALYS and EMPIRE. All the codes describe the shape of the excitation function rather well but the magnitudes are different. It is evident that the scatter in the experimental data is large and more precise measurements are called for.

Despite the great success of the low energy nuclear reactions to produce most of the non-standard positron emitters with high radionuclidic purity, there are several important positron emitters which can be produced on a clinical scale only via intermediate energy chargedparticle beams $(E=40-100 \mathrm{MeV})$. A few examples are: ${ }^{55} \mathrm{Mn}(\mathrm{p}, 4 \mathrm{n}){ }^{52} \mathrm{Fe},{ }^{75} \mathrm{As}(\mathrm{p}, 3 \mathrm{n}){ }^{73} \mathrm{Se},{ }^{85} \mathrm{Rb}(\mathrm{p}, 3 \mathrm{n}){ }^{83} \mathrm{Sr}$, ${ }^{155} \mathrm{Gd}(\mathrm{p}, 4 \mathrm{n}){ }^{152} \mathrm{~Tb}$, etc. The nuclear reaction database of all those processes is rather weak and further experimental and theoretical work is needed.

\subsection{Novel therapeutic radionuclides}

The number of potentially interesting therapeutic radionuclides is very large. However, the emphasis is now on low-range but highly-ionising radiation emitters. In two recent reviews the status of decay and production data of some important emerging therapeutic radionuclides has been discussed [cf. 9, 10]. The scope of this article is therefore limited to only a few important features related to the three groups of radionuclides, namely low-energy $\beta^{-}$ emitters, $\alpha$-emitters and very low-energy electron emitters.

Among the low-energy $\beta^{-}$emitters, ${ }^{47} \mathrm{Sc}\left(\mathrm{T}_{1 / 2}=\right.$ $3.35 \mathrm{~d}),{ }^{67} \mathrm{Cu}\left(\mathrm{T}_{1 / 2}=2.58 \mathrm{~d}\right)$ and ${ }^{186} \operatorname{Re}\left(\mathrm{T}_{1 / 2}=3.78\right.$ d) are of considerable interest. Whereas reliable data are now available for the production of ${ }^{186} \mathrm{Re}$ via the ${ }^{186} \mathrm{~W}(\mathrm{p}, \mathrm{n})$-reaction [for a review cf. 23], for the other two radionuclides the database is weak or rather discrepant. In the case of ${ }^{67} \mathrm{Cu}$, for example, out of the four promising production reactions, namely ${ }^{67} \mathrm{Zn}(\mathrm{n}, \mathrm{p}){ }^{67} \mathrm{Cu}$, ${ }^{68} \mathrm{Zn}(\gamma, \mathrm{p}){ }^{67} \mathrm{Cu},{ }^{70} \mathrm{Zn}(\mathrm{p}, \alpha){ }^{67} \mathrm{Cu}$ and ${ }^{68} \mathrm{Zn}(\mathrm{p}, 2 \mathrm{p}){ }^{67} \mathrm{Cu}$, the fission spectrum averaged (n,p) cross section is known [cf. 24, 25] but it shows some discrepancy with the value integrated from the excitation function. In the case of the $(\gamma, p)$ reaction, only the integral yield has been measured [26]. Among the two charged-particle induced reactions, cross section data of the low energy ${ }^{70} \mathrm{Zn}(\mathrm{p}, \alpha){ }^{67} \mathrm{Cu}$ reaction appear to be reliable but the yield is low and the target material is very expensive. Regarding the ${ }^{68} \mathrm{Zn}(\mathrm{p}, 2 \mathrm{p}){ }^{67} \mathrm{Cu}$ reaction, the yield is high in comparison to all other processes (cf. Fig. 2). However, there is a big discrepancy in the excitation function of the $(\mathrm{p}, 2 \mathrm{p})$ reaction. In view of the increasing significance of ${ }^{67} \mathrm{Cu}$, a precise redetermination of the production data for this reaction is recommended.

Among the group of $\alpha$-emitters for targeted therapy, the radionuclide ${ }^{225} \mathrm{Ac}$ is attracting great attention. Its decay scheme has been recently evaluated [19] and the possible production methods include, (a) separation from nuclear waste, (b) ${ }^{226} \mathrm{Ra}(\mathrm{p}, 2 \mathrm{n}){ }^{225} \mathrm{Ac}$, (c) ${ }^{232} \mathrm{Th}(\mathrm{p}, \mathrm{x}){ }^{225} \mathrm{Ac}$. All the methods are in development. As far as nuclear data are concerned, the database for the $(\mathrm{p}, 2 \mathrm{n})$ reaction is weak 


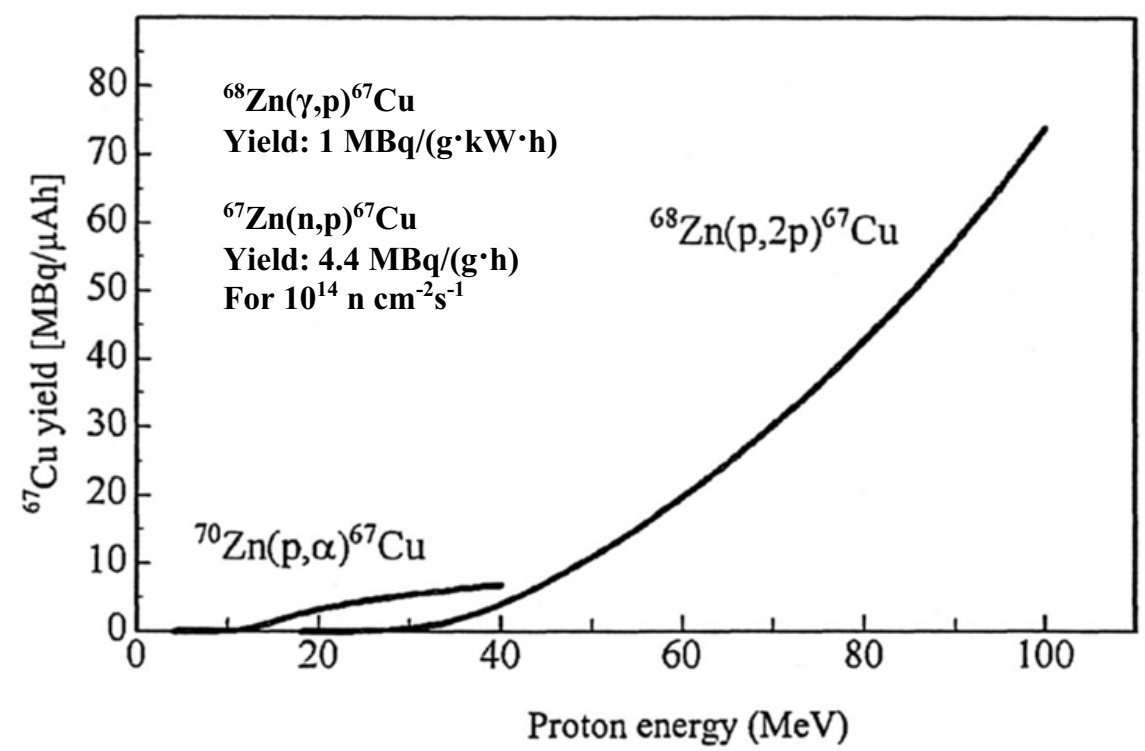

Figure 2. Calculated integral yields of ${ }^{67} \mathrm{Cu}$ in proton-induced reactions on highly enriched target isotopes ${ }^{68} \mathrm{Zn}$ and ${ }^{70} \mathrm{Zn}$, shown as a function of proton energy (curves based on the numerical values given in Ref. [19]). The produc-tion yield for the ${ }^{68} \mathrm{Zn}(\gamma, \mathrm{p})^{67} \mathrm{Cu}$ reaction is taken from Ref. [26] and that for the ${ }^{67} \mathrm{Zn}(\mathrm{n}, \mathrm{p}){ }^{67} \mathrm{Cu}$ reaction from Ref. [25], in both cases using a ${ }^{\text {nat }} \mathrm{Zn}$ target.

[cf. 27]. The spallation of ${ }^{232}$ Th to produce ${ }^{225} \mathrm{Ac}$, however, has been receiving enhanced attention and some detailed measurements have been reported [28,29]. Further work on the formation of impurities is continuing [cf. 30].

As far as the group of very low energy electrons is concerned, three radionuclides, namely ${ }^{117 \mathrm{~m}} \mathrm{Sn}\left(\mathrm{T}_{1 / 2}=\right.$ $13.6 \mathrm{~d}),{ }^{193 \mathrm{~m}} \mathrm{Pt}\left(\mathrm{T}_{1 / 2}=4.33 \mathrm{~d}\right)$ and ${ }^{195 \mathrm{~m}} \mathrm{Pt}\left(\mathrm{T}_{1 / 2}=4.02\right.$ d), are gaining considerable significance. Whereas ${ }^{117 m} \mathrm{Sn}$ emits quasi-monoenergetic conversion electrons, the decay of the other two nuclides leads to the release of an avalanche of Auger electrons. All the three radionuclides constitute a high-spin isomeric state of the respective isotope. As discussed recently in detail [31], such states are preferentially populated in $\alpha$-particle induced reactions. Some production data for the three radionuclides are available [cf. 32-35] but the database needs further improvement. More detailed information on the Auger electron spectra is also needed.

\section{Concluding remarks and future data needs}

The foregoing discussion shows that in external radiation therapy, the atomic and molecular data are of paramount importance. Nuclear data play a very important role in the development of radionuclides for in-vivo applications.

The radioactive decay data govern the choice of a radionuclide for medical application and the nuclear reaction data allow optimisation of its production route. Whereas the data needed in routine patient-care studies are available and are generally sufficient, the development of non-standard positron emitters and novel low-range highly-ionising radiation emitters for internal radiotherapy demands considerable amount of new and reliable decay and reaction data. The former are generally produced at a low-energy $(\mathrm{E}<20 \mathrm{MeV}$ ) cyclotron via the low-energy $(\mathrm{p}, \mathrm{n})$ reaction. However, for the production of several positron emitters, like ${ }^{52} \mathrm{Fe}$ and ${ }^{73} \mathrm{Se}$, and of therapeutic radionuclides such as ${ }^{67} \mathrm{Cu}$ and ${ }^{225} \mathrm{Ac}$, intermediate energy protons from a cyclotron or an accelerator are needed. The $\alpha$-particle beam is also very useful in populating lowlying high-spin isomeric states which deexcite by IT and emit low-energy electrons (conversion or Auger, or both) that can be used in internal therapy. Examples are ${ }^{117 \mathrm{~m}} \mathrm{Sn}$, ${ }^{193 \mathrm{~m} P t},{ }^{195 \mathrm{~m} P t}$, etc..

The intermediate-term nuclear data needs are summarized below.

Non-standard $\beta^{+}$emitters

- Re-evaluate existing mass decay chains

- Determine $\beta^{+}$emission intensities (using improved experimental techniques)

- Evaluate existing charged-particle reaction data

- Strengthen database via measurements and calculations

- Validate evaluated data through integral yield measurements.

\section{Novel therapeutic radionuclides}

- Re-evaluate intensities of emitted corpuscular radiation

- Improve knowledge of Auger electron spectra

- Strengthen charged-particle database for production (via measurements and calculations).

Over the last few years, two important new developments in the application of radionuclides have been emerging. The first one deals with a combination of PET and internal radiotherapy (theranostic approach) and the second one with a combination of PET and magnetic resonance imaging (MRI). In the former case a $\beta^{+}$emitting and a therapeutic radionuclide of the same element are simultaneously injected in a patient, as it was originally done using ${ }^{86} \mathrm{Y}$ and ${ }^{90} \mathrm{Y}$ [36]. In the latter case a $\beta^{+}$ emitting radionuclide of the contrast agent manganese [e.g. $\left.{ }^{52} \mathrm{Mn}\left(\mathrm{T}_{1 / 2}=5.6 \mathrm{~d}\right)\right]$ is useful. Evidently, for those two fast expanding applications, further novel radionuclides will have to be developed, entailing also detailed nuclear data studies. On a longer term basis, the radionuclide research 
could also involve a combination of radioactivity and nanotechnology, provided toxicity problems are overcome. The future perspectives of radionuclide research for medical applications, involving also nuclear data studies, thus appear to be very bright.

Development work is also underway on newer irradiation technologies for medical radionuclide production, e.g. intermediate energy charged-particle accelerators, highpower electron accelerators for photon production, and spallation neutron sources. The supporting nuclear data research will therefore also continue to receive further attention [cf. 10].

The author thanks Prof. B. Neumaier for his support of the Radionuclide Research Programme at Jülich, and Dr. B. Scholten for critical discussions.

\section{References}

[1] S.M. Qaim (Editor) Nuclear data for medical applications, Special Issue of the journal Radiochim. Acta 89, 189-355 (2001)

[2] Atomic and molecular data for radiotherapy and radiation research (IAEA-TECDOC-799, Vienna, 1995)

[3] H.H. Barschall, M.B. Chadwick, D.T.L. Jones, J.P. Meulders, H. Schumacher, P.G. Young, Nuclear data for neutron and proton radiotherapy and for radiation protection (ICRU Report 63, Bethesda, 2000)

[4] K. Kettern, H.H. Coenen, S.M. Qaim, Radiation Phys. Chem. 78, 380 (2009)

[5] G. Stöcklin, S.M. Qaim, F. Rösch, Radiochim. Acta 70/71, 249 (1995)

[6] S.M. Qaim, Radiochim. Acta 89, 223 (2001)

[7] Charged particle cross-section database for medical radioisotope production: diagnostic radioisotopes and monitor reactions (IEAE-TECDOC-1211, Vienna, 2001)

[8] K.F. Eckerman, A. Endo, Radioactive decay data and decay schemes (SNM-MRID Committee, Reston, Va, USA, 2007)

[9] S.M. Qaim, J. Radioanal. Nucl. Chem. 305, 233 (2015)

[10] S.M. Qaim, Nucl. Med. Biol. 44, 31 (2017)

[11] S.M. Qaim, Nucl. Phys. A 185, 614 (1972)

[12] Y. Nagai, Y. Hatsukawa, J. Phys. Soc. Jpn. 78, 033201 (2009)

[13] R. Crasta, H. Naik, S.V. Suryanarayana, P.M. Prajapati, K.C. Jagadisan, S.V. Thakara, S. Ganesh, V.T. Nimje, K.C. Mittal, A. Goswami, J. Radioanal. Nucl. Chem. 290, 367 (2011)

[14] O. Lebeda, M. Pruszynski, Appl. Radiat. Isot. 68, 2355 (2010)

[15] K. Gagnon, F. Bérnard, M. Kovacs, T.J. Ruth, P. Schaffer, J.S. Wilson, S.A. McQuarrie, Nucl. Med. Biol. 38, 907 (2011)
[16] F. Tárkányi, F. Ditroi, A. Hermanne, S. Takács, A.V. Ignatyuk, Nucl Instrum. Meth. B. 280, 45 (2012)

[17] S.M. Qaim, S. Sudar, B. Scholten, A.J. Koning, H.H. Coenen, Appl. Radiat. Isot. 85, 101 (2014)

[18] S.M. Qaim, Radiochim. Acta 89, 297 (2001)

[19] S.M. Qaim, F. Tárkányi, R. Capote (Editors), Nuclear data for the production of therapeutic radionuclides (Technical Reports Series No. 473, IAEA, Vienna, 2011)

[20] S.M. Qaim, Radiochim. Acta 99, 611 (2011)

[21] M.N. Aslam, S. Sudár, M. Hussain, A.A. Malik, H.A. Shah, S.M. Qaim, Radiochim. Acta 97, 669 (2009)

[22] H. Zaneb, M. Hussain, N. Amjed, S.M. Qaim, Appl. Radiat. Isot. 104, 232 (2015)

[23] M. Hussain, S. Sudár, M.N. Aslam, A.A. Malik, R. Ahmad, S.M. Qaim, Radiochim. Acta 98, 385 (2010)

[24] A. Calamand, in Handbook on nuclear activation cross sections (Technical Report No. 156, IAEA, Vienna, 1974; pp. 273-324)

[25] M.S. Uddin, M.R. Zaman, S.M. Hossain, S.M., Qaim, Radiochim. Acta 102, 473 (2014)

[26] V.N. Starovaitova, L. Tchelidze, D.P. Wells, Appl. Radiat. Isot. 85, 39 (2014)

[27] C. Apostolidis, R. Molinet, G. Rasmussen, A. Morgenstern, Appl. Radiat. Isot. 62, 383 (2005)

[28] S.V. Ermolaev, B.L. Zhuikov, V.M. Kokhanyuk, V.L. Matushko, S.N. Kalmykov, R.A. Aliev, I.G. Tananaev, B.F. Myasoudov, Radiochim. Acta 100, 223 (2012)

[29] J.W. Weidner, S.G. Maschnik, K.D. John, F. Hemez, B.D. Ballard, H. Bach, E.R. Birnbaum, L.J. Bitteker, A. Couture, D. Dry, M.E. Fassbender, F.M. Nortier, Appl. Radiat. Isot. 70, 2602 (2012).

[30] J.W. Engle, J.W. Weidner, B.D. Ballard, M.E. Fassbender, L.A. Hudston, K.R. Jackman, D.E. Dry, L.E. Wolfsberg, L.J. Bitteker, J.L. Ullman, M.S. Gulley, C. Pillai, G. Goff, E.R. Birnbaum, K.D. John, S.G. Mashnik, F.M. Nortier, Radiochim. Acta 102, 569 (2014)

[31] S.M. Qaim, I. Spahn, B. Scholten, B. Neumaier, Radiochim. Acta 104, 601 (2016)

[32] S.M. Qaim, H. Döhler, Int. J. Appl. Radiat. Isot. 25, 645 (1984)

[33] R. Adam Rebeles, A. Hermanne, P. van den Winkel, F. Tárkányi, S. Takács, L. Daraban, Nucl. Instr. Meth. B 266, 4731 (2008)

[34] K. Hilgers, H.H. Coenen, S.M. Qaim, Appl. Radiat. Isot. 66, 545 (2008)

[35] M.S. Uddin, B. Scholten, A. Hermanne, S. Sudár, H.H. Coenen, S.M. Qaim, Appl. Radiat. Isot. 68, 2001 (2010)

[36] H. Herzog, F. Rösch, G. Stöcklin, C. Lueders, S.M. Qaim, L.E. Feinendegen, J. Nucl. Med. 34, 2222 (1993) 